\title{
A Pilot Study of Serum Parathyroid Hormone in Acute Coronary Syndrome
}

\author{
MD. HARISUL HOQUE, SM MUSTAFA ZAMAN, KHURSHID AHMED, \\ MD. FAISAL IBNE KABIR, NILUFAR FATEMA \\ Department of Cardiology, Bangabandhu Sheikh Mujib Medical University, Dhaka. \\ Address of Correspondence : Dr. Nilufar Fatema, Consultant, Department of Cardiology, Bangabandhu Sheikh Mujib Medical \\ University, Dhaka.Email: nilufar.fatema@gmail.com
}

\begin{abstract}
:
Acute coronary syndrome (ACS) is the public health issue as it is the single most common cause of death in the world. Role of vitamin D in cardiovascular health is of much interest at present. Experimental, as well as, some observational studies suggest that vitamin $D$ and its metabolites are integrally related to blood pressure and the rennin-angiotensin system. Vitamin D insufficiency affects almost 50\% of the population worldwide. Few studies have been carried out to determine the prevalence of hypovitaminosis D in Bangladesh. 30 Patient with ACS were included in this study who had admitted in the department of Cardiology of Bangabandhu Sheikh Mujib Medical University (BSMMU) patient with in first 48 hours of the onset of chest pain. This study was conducted from $1^{\text {st }}$ July'18 to $30^{\text {th }}$ June'19 (One year) by Purposive sampling. Data analysis was carried out by using the statistical package for Social Science (SPSS) version 20.0 windows software. Baseline characteristics have been compared using the independent sample student t-test test where appropriate. The student t-test was used to assess the correlation between PTH levels. p-value $<0.05$ was taken as significant. We found an association between elevated PTH levels and ACS.
\end{abstract}

Key Words: ACS, PTH

University Heart Journal 2020; 16(2): 71-77

\section{Introduction:}

Acute coronary syndrome (ACS) is the public health issue as it is the single most common cause of death in the world. The frequency of this disease is increasing. ACS accounted for approximately 1.1 million discharges in the United States in 2009. However, in Europe there has been an overall trend for a reduction in Ischaemic heart disease (IHD) mortality during the past three decades. IHD accounts for almost 1.8 million annual deaths or $20 \%$ of all deaths in Europe, although with large variations between countries. The relative incidences of STEMI are decreasing and NSTEMI are increasing. There is a consistent pattern for STEMI to be relatively more common in younger than in order people and more common in men than in women. In several recent studies have been highlighted a fall in acute and long-term mortality following STEMI in parallel with greater use of reperfusion therapy, primary percutaneous coronary intervention (PCI), modern antithrombotic therapy, and secondary prevention. Still, mortality remains substantial: the in-hospital mortality of unselected patients with STEMI in the national registries of European countries varies between $4 \%$ and $12 \%$, while reported one year mortality among STEMI patients in angiography registries is approximately $10 \%$. ACS occurs three to four times more often in men than in women below the age of 60 , but after the age of 75 , women represent the majority of patients.

Bangladesh has been experiencing epidemiological transition from communicable disease to noncommunicable disease (NCD). The overall mortality rate has decreased significantly over the last couple of decades. But deaths due to chronic diseases, specially the 'fatal four' i.e. cardiovascular disease (CVD), cancer, chronic respiratory disease and diabetes, are increasing in an alarming rate. ${ }^{1} \mathrm{CAD}$ is an important contributor to one of the four i.e. CVD. Of all South Asian countries, Bangladesh probably has the highest rates of CVD and yet is the least studied; in the global combat against CVD, Bangladesh is a country 'missing in action'. ${ }^{2}$ Besides epidemiological transition, widespread environmental contaminants such as arsenic in groundwater or particulate matter in air, or specific vulnerabilities in the genetic or metabolic make-up, may play important role in the etiopathogenesis of CAD in this population. Genetic predisposition, high prevalence of metabolic syndrome and conventional risk factors plays important role. 
Lifestyle related factors, including poor dietary habits, excess saturated and trans fat, high salt intake, and lowlevel physical activity may be important as well. Some novel risk factors, including hypovitaminosis $\mathrm{D}$, arsenic contamination in water and food-stuff, particulate matter air pollution may play unique role. At the advent of the new millennium, we know little about our real situation. Large scale epidemiological, genetic and clinical researches are needed to explore the different aspects of CAD in Bangladesh. The prevalence of CAD varies considerably by populations, may be up to 10 folds. ${ }^{3}$ South Asians are unduly prone to develop CAD. ${ }^{4}$ Most notable features of $\mathrm{CAD}$ in this population are the extreme prematurity and severity; 2-4-fold higher prevalence, incidence, hospitalization and mortality; 5-10 years earlier onset of first myocardial infarction (MI) and 5-10-fold higher rates of MI and death before the age of 40 years. ${ }^{4}$ The exact prevalence of CAD in Bangladesh is not known. Only a limited number of small-scale epidemiological studies are available. Probably the prevalence of IHD was first reported in 1976, which was $0.33 \% .^{5}$ More recent data indicates CAD prevalence between $1.85 \%{ }^{6}$ and $3.4 \%{ }^{7}$ in rural and $19.6 \%$ in an urban sample of working professionals. ${ }^{8,9}$ Despite marked disparity in values, there seems to be a rising prevalence of CAD in Bangladesh. A recent study from rural Bangladesh demonstrated a dramatic increase in CVD from 1986 to 2006. The age-standardized CVD mortality rates was increased by 30-fold (from 16 deaths per 100,000 to 483 deaths per 100,000) among males and 47-fold (from 7 deaths per 100,000 to 330 deaths per 100,000 ) in females. ${ }^{10}$ A nation-wide survey is needed to find out the current epidemiological aspects of CAD in the country.

Studies involving the immigrants in abroad have found that among the South Asians, Bangladeshis are even more prone to develop CAD, and are associated with higher morbidity and mortality related to CAD. Bangladeshis in New York, USA, had more extensive and severe heart disease with 53\% having triple-vessel disease compared to $26 \%$ among whites. ${ }^{11}$

Role of vitamin D in cardiovascular health is of much interest at present. Experimental, as well as, some observational studies suggest that vitamin D and its metabolites are integrally related to blood pressure and the rennin-angiotensin system. Vitamin D insufficiency affects almost $50 \%$ of the population worldwide. Few studies have been carried out to determine the prevalence of hypovitaminosis D in Bangladesh. High prevalence of suboptimal serum 25-hydroxycholecalciferol levels
$(<25 \mathrm{nmol} / \mathrm{l})$ was described in lactating women of low socioeconomic status and those wearing Shari, a traditional ladies wear. ${ }^{12}$ In another survey of women aged 18-60 years, serum 25-hydroxycholecalciferol levels were $<40 \mathrm{nmol} / 1$ in $78 \%$ of university students and $83 \%$ of veiled women. ${ }^{13}$ Betel nut chewing, which is prevalent in Bangladesh, has been reported to contribute to hypovitaminosis $\mathrm{D}$ by modulating the enzymes regulating circulating 1,25-dihydroxycholecalciferol concentration. ${ }^{14}$ Positive correlation has been found between vitamin $\mathrm{D}$ deficiency and reduced level of apoA1 in Bangladeshi people in UK independent of glycemia and other dietary, anthropometric, and lifestyle related risk factors for type $2 \mathrm{DM}$ and IHD. ApoA1 deficiency may in turn lead to low level of HDL, thereby increasing the risk of CAD. ${ }^{15}$ An association between vitamin $\mathrm{D}$ receptor polymorphisms and insulin secretory capacity has been described among Bangladeshi Asians in UK. ${ }^{16}$ Further researches are needed to find out the association, if any, between vitamin D deficiency and CAD in Bangladesh.

Elevated PTH levels ( $>55 \mathrm{pg} \mathrm{ml} / \mathrm{L}$ ) were seen in $16 \%$ of the patients, being most frequent in patients with myocardial infarction (28\%) and congestive heart failure (42\%). The levels were significantly correlated with the APACHE II score $(\mathrm{r}=0.48, \mathrm{P}<0.0001)$ and with the length of stay in hospital $(\mathrm{r}=0.26, \mathrm{p}<0.002)$. PTH was also significantly $(\mathrm{p}<0.03)$ elevated in non-survivors compared with survivors and was found to be a stronger predictor of mortality $(\mathrm{p}<0.01)$ than the APACHE II score $(\mathrm{P}<0.02$ in Cox's proportional hazard analysis. ${ }^{17}$ Gang-Yong Wu et al. found in one study in one study that the serum PTH levels correlated with Coronary artery calcification (CAC) and may thus be used as a reliable predictor of CAC in patients without renal failure. ${ }^{18}$ The serum PTH levels were significantly raised in the AMI patients and did not normalize during the observation time. As the PTH concentrations were unexplained by infarct size, concomitant disease or medication, so their finding suggests that PTH could be an independent risk factor for AMI. ${ }^{19}$ It has also been suggested that elevated PTH levels may be related to nonfatal atherosclerotic CVD and development of atherosclerosis in peripheral and central large arteries. ${ }^{20}$ In a recent study, Shekarkhar et al. demonstrated elevated PTH levels have been associated with cardiac contractility, cardiomyocyte hypertrophy, and apoptosis, as well as structural and functional changes in the vascular system and low left-ventricular ejection fraction. And there is an association between PTH levels and the number of stenotic coronary arteries. This study also showed that patients with high PTH levels were more hypertensive and had lower ejection fraction. ${ }^{21}$ 


\section{Ethical Issues:}

Ethical clearance has been taken from the institutional review board of BSMMU. The study was carried out according to Helsinki Declaration for Medical Research involving Human subjects 1964. Participants have been informed in detail about the nature and purpose of the study. It was a non-interventional study. There was no physical, psychological and social risk to the subject. Each participant has been included according to their wish to participate. They were free to withdraw their participation at any stage of the study. Data taken from the participants were regarded as confidential and has kept locked under principal investigator. Data has been used only for this scientific study. Informed written consent was taken from each participant.

\section{Materials and Methods:}

\section{Study population:}

30 Patient with ACS were included in this study who had admitted in the department of Cardiology of Bangabandhu Sheikh Mujib Medical University (BSMMU) patient within first 48 hours of the onset of chest pain.

\section{Study design:}

Cross sectional observational study

Study period:

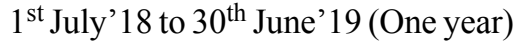

\section{Place of Study:}

This study had done in the 'Heart Failure Division' Department of Cardiology, BSMMU, Shahbagh, Dhaka, Bangladesh.

\section{Sampling Technique:}

Purposive sampling

\section{Sample Size:}

30 eligible patients had included under study.

\section{Operational Definitions:}

\section{A. Acute coronary syndrome: ${ }^{22}$}

The leading symptom that initiates the diagnostic and therapeutic cascade in patients with suspected acute coronary syndromes (ACS) is chest pain. Based on the electrocardiogram (ECG), two groups of patients should be differentiated. The clinical spectrum of non-STelevation ACS (NSTE-ACS) may range from patients free of symptoms at presentation to individuals with ongoing ischaemia, electrical or haemodynamic instability or cardiac arrest. The pathological correlate at the myocardial level is cardiomyocyte necrosis [NSTE-myocardial infarction (NSTEMI)] or, less frequently, myocardial ischaemia without cell loss (unstable angina). A small proportion of patients may present with ongoing myocardial ischaemia, characterized by one or more of the following: recurrent or ongoing chest pain, marked ST depression on 12-lead ECG, heart failure and haemodynamic or electrical instability. Due to the amount of myocardium in jeopardy and the risk of malignant ventricular arrhythmias, immediate coronary angiography and, if appropriate, revascularization are indicated.

1. Patients with acute chest pain and persistent $(>20$ min) ST-segment elevation.

This condition is termed ST-elevation ACS and generally reflects an acute total coronary occlusion. Most patients will ultimately develop an ST-elevation myocardial infarction (STEMI). The mainstay of treatment in these patients is immediate reperfusion by primary angioplasty or fibrinolytic therapy. ${ }^{1}$

2. Patients with acute chest pain but no persistent STsegment elevation.

ECG changes may include transient ST-segment elevation, persistent or transient ST-segment depression, T-wave inversion, flat $\mathrm{T}$ waves or pseudonormalization of $\mathrm{T}$ waves or the ECG may be normal.

B. Diabetes:

$\left(\right.$ American Diabetes association 2017) ${ }^{23}$

History of diagnosis and /or on treatment of diabetes by a physician

The American Diabetes Association 2017 criteria include documentation of following:

1. Fasting plasma glucose $>126 \mathrm{mg} / \mathrm{dl}(7.0 \mathrm{mmol} / \mathrm{l})$

2. 2 hours plasma glucose $>200 \mathrm{mg} / \mathrm{dl}(11.1 \mathrm{mmol} / \mathrm{l})$ during an oral glucose tolerance test $(75 \mathrm{~g})$ or

3. Haemoglobin Alc $>6.5 \%(48 \mathrm{mmol} / \mathrm{l})$

4. In a patient with classic symptoms of hyperglycemia or hyperglycemic crisis, a random plasma glucose $>200 \mathrm{mg} / \mathrm{dl}(11.1 \mathrm{mmol} / \mathrm{l})$

C. Hypertension :

(The $7^{\text {th }}$ reports of JNC 2003) $)^{24}$

Documented by,

1. History of hypertension diagnosed and treated with medication, diet and/or exercise. 
2. Systolic blood pressure $>140 \mathrm{~mm}$ of $\mathrm{Hg}$ and/or diastolic blood pressure $>90 \mathrm{~mm}$ of $\mathrm{Hg}$ on at least two occasions.

3. The patient is on antihypertensive drug treatment.

D. Coronary Artery disease: 25

Will be considered to be present if there is history of Chronic angina, a stress test positive for ischaemia, a prior myocardial infarction, coronary stenosis $>50 \%$ by cardiac catheterization, or prior coronary angioplasty or coronary bypass surgery.

E Dyslipidaemia: ${ }^{26}$

Indicate if the patient has a history of dyslipidaemia that was diagnosed and/or treated by a physician. National Cholesterol Education Program (NCEP) criteria include documentation of following: Total Cholesterol $>200 \mathrm{mg} / \mathrm{dl}(5.18 \mathrm{mmol} / \mathrm{l})$

Low density lipoprotein Cholesterol (LDL_C) $>130$ $\mathrm{mg} / \mathrm{dl}(3.37 \mathrm{mmol} / \mathrm{l})$

High density lipoprotein Cholesterol (HDL_C) $<40$ $\mathrm{mg} / \mathrm{dl}(1.04 \mathrm{mmol} / \mathrm{l})$ in men and $<50 \mathrm{mg} / \mathrm{l}(1.3 \mathrm{mmol} / \mathrm{l})$ in women

Currently receiving antilipidemic treatment

F. Smoking: 26

Current: smoking cigarettes within 1 month of admission

Recent stopped smoking between 1 month and 1 year of admission

Past : Stopped smoking $>1$ year ago

Never: Never smoked

G. Ejection Fraction: ${ }^{27}$

Left Ventricular Ejection fraction is a measurement of how much blood the left ventricular pumps out with each Cardiac cycle.

A normal heart's ejection fraction is $>55 \%$

It is calculated as the difference between LV enddiastolic volume (EDV) and end-systolic volume (ESV) divided by LV end-diastolic volume (EDV)

$\mathrm{LVEF}=$ Stroke volume $(\mathrm{EDV}-\mathrm{ESV}) / \mathrm{EDV}$

It remains the most commonly used method for assessing systolic function.

\section{Study Procedure:}

This cross-sectional observational study has conducted in cardiology department of BSMMU from July 2018 to
June 2019 after receiving approval of the protocol by Institutional Review Board (IRB) of Bangabandhu Sheikh Mujib Medical University (BSMMU). Patients with Acute coronary syndrome who had admitted in Cardiology department of BSMMU within 48 hours of the onset of chest pain enrolled into this study after fulfilling the inclusion and exclusion criterias. The purposes of the study have been explained in details to each subject and inform written consent was obtained. After getting consent, meticulous history was taken and relevant clinical examination was performed and recorded in predesigned structural proforma. Demographic data (Age, sex), risk factor profile (DM, HTN, BMI, Dyslipidaemia, Smoking, Family history of CAD) were recorded. 12 lead ECG had done to detect any ST change or new onset LBBB. During admission baseline investigations such as Troponin-I and CKMB 4 sample. If first sample showed increase titre then second sample was not taken. Blood for CBC, CRP, Blood sugar- FBS, 2 Hr ABF, HbA1c, (F) Lipid profile, Serum Urea, Serum creatinine, Serum electrolyte, S. Uric acid, S. Bilirubin, SGPT, SGOT, S.Alk. Phos, S.Total Protein, S. Albumin, A/G ratio, TSH, FT3, FT4 were done to detect risk factors and secondary causes. Xray chest P/A view was needed to see any cardiomegaly. Urine(R/E) was also done. If evidence of MI or UA then another blood sample was taken to do PTH level, $\mathrm{Ca}++$ level within the first 48 hours of the onset chest pain. 2D and M-mode Echocardiography was done to evaluate the regional wall motion abnormality, LV systolic and diastolic function and RV systolic function, to detect any mechanical complication of MI. USG of thyroid was done to detect any pathology in thyroid and Parathyroid scan was done to exclude any parathyroid adenoma. BMD was done to see bone mineral density.

\section{Selection criteria of patient:}

\section{Inclusion Criteria:}

1. Patients 18 years of age or above.

2. Patients present within 48 hours of the onset of chest pain.

3. Acute coronary syndrome patients (UA, NSTEMI, STEMI)

4. Give inform written consent.

\section{Exclusion Criteria:}

i) Chronic kidney disease patients

ii) Deformed bony disease

iii) Osteoporosis

iv) Patient in steroid. 


\section{Statistical Methods:}

\section{Data collection:}

Data was collected according to the predesigned semi structured data collection sheet.

Data Analysis:

Statistical analyses was done and reviewed by the investigators. After editing data analysis was carried out by using the statistical package for Social Science (SPSS) version 20.0 windows software. Baseline characteristics have been compared using the independent sample student t-test test where appropriate. The student t-test was used to assess the correlation between PTH levels. p-value $<0.05$ was taken as significant.

\section{Results:}

Total 30 patients of acute coronary syndrome (ACS) were included in this study. ACS was confirmed by ECG change, cardiac bio markers (CKMB and Troponin- I). PTH level was high in $22(73 \%)$ patients, low in $3(10 \%)$ and normal in 5 (16\%) patients. Patients who have high PTH, 13(59\%) patients had STEMI, $6(27 \%)$ had NSTEMI and 3 (13\%) had unstable angina. High serum PTH was seen more in acute STEMI cases than NSTEMI or UA. 5 patients have normal PTH and only 3 patients have low PTH.

Table-I

Distribution of parathyroid status $\quad(n=30)$

\begin{tabular}{lcc}
\hline Parathyroid status & Frequency & Percentage \\
\hline High & 22 & 73.0 \\
Normal & 5 & 16.0 \\
Low & 3 & 10.0 \\
\hline
\end{tabular}

Table-II

Distribution of parathyroid status according to type of $A C S$

\begin{tabular}{|c|c|c|c|c|c|}
\hline \multirow[t]{2}{*}{ Type of ACS } & \multicolumn{2}{|c|}{$\begin{array}{c}\text { High } \\
\text { PTH } \\
(\mathrm{n}=22)\end{array}$} & \multicolumn{2}{|c|}{$\begin{array}{c}\text { Normal or } \\
\text { Low PTH } \\
\quad(n=8)\end{array}$} & \multirow[t]{2}{*}{$P$ value } \\
\hline & No & $(\%)$ & No & $(\%)$ & \\
\hline STEMI & 13 & 59.0 & 2 & 25 & 0.005 \\
\hline NSTEMI & 6 & 27.0 & 4 & 50 & 0.763 \\
\hline Unstable angina & 3 & 13.0 & 2 & 25 & 0.655 \\
\hline
\end{tabular}

\section{Discussion:}

This study demonstrated that there is association between PTH levels and ACS. On the other hand, there was a statistically significant but weak correlation between PTH levels and Gensini score. It is well known that PTH regulates blood calcium and phosphorus concentrations. Elevated calcium is a risk factor for vascular disease, and it is associated with increased vascular stiffness and high pulse pressure. ${ }^{28}$ Previous studies demonstrated that elevated PTH levels are related to impaired vascular function, increased vascular stiffness, carotid and brachial artery intima-media thickness, and increased systolic and diastolic blood pressure. ${ }^{28-30}$ Previous studies showed that increased levels of PTH are associated with high risks for cardiovascular mortality and morbidity. ${ }^{31}$ It has also been suggested that elevated PTH levels may be related to nonfatal atherosclerotic CVD and development of atherosclerosis in peripheral and central large arteries. ${ }^{32}$ Moreover, elevated PTH levels have been associated with cardiac contractility, cardiomyocyte hypertrophy, and apoptosis, as well as structural and functional changes in the vascular system and low left-ventricular ejection fraction. ${ }^{33-38}$ Additionally, elevated circulating levels of PTH are associated with significantly decreased functional capacity and advanced heart failure. ${ }^{39}$

According to recent data, PTH is suggested to affect the cardiovascular system through various mechanisms. ${ }^{33-37}$ Many studies investigated the relationship between CAD and PTH, but conflicting results were obtained. ${ }^{40-42} \mathrm{We}$ found a similar result in our study population with ACS who has investigated. Shekarkhar et al. demonstrated an association between PTH levels and the number of stenotic coronary arteries. This study also showed that patients with high PTH levels were more hypertensive and had lower ejection fraction. ${ }^{43}$ Our results were inconsistent with the findings of this study. We did not find significant differences between PTH levels and the number of stenotic coronary arteries or ejection fraction or a correlation between PTH level and Gensini score. We also could not demonstrate an association between PTH level and ACS or its subgroups. The strict exclusion criteria for our study population may have influenced the results. Moreover, we accept that our study population and groups were not homogeneous; but when we evaluated the relationship between the groups separately, the results did not change. However, more accurate results could be obtained by evaluating patients according to their glomerular filtration rate and PTH levels. The relationship between PTH and CVD was shown particularly in patients with chronic renal disease. ${ }^{39}$ Also, higher PTH levels may be related with the risk of sudden cardiac death in elderly populations without CVD. ${ }^{44}$ Our study was a cross-sectional study and has no long-term mortality or morbidity data; therefore, our results cannot provide additional data on this issue. 


\section{Conclusion:}

It is difficult to draw a simple conclusion regarding the linear relationship between Acute Coronary Syndrome with raised Paratharmone level. However multicentre study with enrolment of different races are deemed to necessary before making a final comment.

\section{Declaration of interests:}

This project has been done with the fund from the Ministry of Science \& Technology, Government of the Peoples Republic of Bangladesh for the Fiscal year 2018-19.

\section{Acknowledgements:}

The authors would like to thank the patients who participated in this study and their family members, as well as other investigator and stuff at all of Cardiology, biochemistry department of BSMMU.

\section{Reference:}

1. Strategic Plan for Surveillance and Prevention of Noncommunicable Diseases in Bangladesh 2011-2015. Directorate General of Health Services, Ministry of Health and Family Welfare; Dhaka: August 2011; p-7.

2. Department of Public Health and Primary Care, University of Cambridge. High-risk Hearts: A South Asian Epidemic. [homepage on the internate] c2013 [cited 2013 July 3].

3. Levy D., Kannel W.B. Searching for answers to ethnic disparities in cardiovascular risk. Lancet. 2000;356:266-267.

4. Enas E.A., Senthilkumar A. Coronary artery disease in Asian Indians: an update and review. Int J Cardiol. 2001;1(2)

5. Malik A. Congenital and acquired heart diseases (a survey of 7062 persons) Bangladesh Med Res Counc Bull. 1976;II: 115-119.

6. Sayeed M.A., Mahtab H., Sayeed S. Prevalence and risk factors of coronary heart disease in a rural population of Bangladesh. Ibrahim Med Coll J. 2010;4:37-43.

7. Zaman M.M., Ahmed J., Choudhury S.R. Prevalence of ischemic heart disease in a rural population of Bangladesh. Indian Heart J. 2007;59:239-241.

8. Ahsan S., Haque K.M.H.S., Salman M. Detection of ischaemic heart disease with risk factors in different categories of employees of University Grants Commission. Univ Heart J. 2009;5:20-23.

9. Saquib N., Saquib J., Ahmed T. Cardiovascular diseases and type 2 diabetes in Bangladesh: a systematic review and metaanalysis of studies between 1995 and 2010. BMC Public Health. 2012;12:434.

10. AhsanKarar Z., Alam N., Kim Streatfield P. Epidemiological transition in rural Bangladesh, 1986-2006. Glob Health Action. 2009:2.

11. Silbiger J.J., Ashtiani R., Attari M. Atherosclerotic heart disease in Bangladeshi immigrants: risk factors and angiographic findings. Int J Cardiol. 2011;146:e38-40.
12. Islam M.Z., Lamberg-Allardt C., Kärkkäinen M. Vitamin D deficiency: a concern in premenopausal Bangladeshi women of two socio-economic groups in rural and urban region. Eur $\mathrm{J}$ Clin Nutr. 2002;56:51-56.

13. Islam M.Z., Akhtaruzzaman M., Lamberg-Allardt C. Hypovitaminosis D is common in both veiled and non-veiled Bangladeshi women. Asia Pac J Clin Nutr. 2006;15:81-87.

14. Ogunkolade W.B., Boucher B.J., Bustin S.A. Vitamin D metabolism in peripheral blood mononuclear cells is influenced by chewing "betel nut" (Areca catechu) and vitamin D status. J Clin Endocrinol Metab. 2006;91:2612-17.

15. John W.G., Noonan K., Mannan N. Hypovitaminosis D is associated with reductions in serum apolipoprotein A-I but not with fasting lipids in British Bangladeshis. Am J Clin Nutr. 2005;82:517-22.

16. Ogunkolade B.W., Boucher B.J., Prahl J.M. Vitamin D receptor (VDR) mRNA and VDR protein levels in relation to vitamin D status, insulin secretory capacity, and VDR genotype in Bangladeshi Asians. Diabetes. 2002;51:2294-2312.

17. Carlstedt F, et al. Serum levels of parathyroid hormone are related to the mortality and severity of illness in patients in the emergency department. Eur J Clin Invest. 1997 Dec; 27 (12): 982-83.

18. Gang- Yong $\mathrm{Wu}$ et al. Correlation between serum parathyroid hormone levels and coronary artery calcification in patients without renal failure.

19. Ljunghall S, et al. Serum electrolytes and parathyroid hormone concentrations in acute myocardial infarction. Exp Clin Endocrinol. 1986 Nov; 88 (1): 95-100.

20. Hagström E, Michaëlsson K, Melhus H, Hansen T, Ahlström $\mathrm{H}$, Johansson L, et al. Plasma parathyroid hormone is associated with subclinical and clinical atherosclerotic disease in 2 community-based cohorts. Arterioscler Thromb Vasc Biol. 2014;34:1567-73.

21. Shekarkhar S, Foroughi M, Moatamedi M, Gachkar L. The association of serum parathyroid hormone and severity of coronary artery diseases. Coron Artery Dis. 2014;25: 339-42.

22. Marco Rofi et al. 2015 ESC Guidelines for the management of acute coronary syndromes in patients presenting without persistent ST-segment elevation: Task Force for the Management of Acute Coronary Syndromes in Patients Presenting without Persistent ST-Segment Elevation of the European Society of Cardiology (ESC) European Heart Journal, 2016; 37(3): 267-315.

23. American Diabetes Association 'Standards of Medical Care in Diabetes-2017’, Diabetes Care, 2017;40(suppl. 1):1-135.

24. Chobanian AV, Bakris GL, Black HR, Cushman WC, Green LA \& Izzo JL, et al. The Seventh Report of the Joint National Committee on Prevention, Detection, Evaluation and Treatment of High Blood Pressure: the JNC 7 report', JAMA, 2003;289(19):2560-72.

25. Klapholz M, Maurer M, Lowe AM, Messineo F, Meisner JS \& Mitcell $\mathrm{M}$ et al. 'Hospitalization for Heart Failure in the 
Presence of a Normal Left Ventricular Ejection Fraction: Results of the New York Heart Failure Registry', J Am Coll Cardiol, 2004;43(8):1432-38.

26. Cannon CP, Brindis RJ, Chaitmen BR, Cohen DJ \& Cross T et al. '2013 ACCF/AHA Key Data elements and Definitions for Measuring the clinical management and Outcomes of Patients With Acute Coronary Syndrome and Coronary Artery Disease', Circulation, 2013;127:1052-89.

27. Solomon, SD, Wu, J \& Gillam, L. 'Echocardiography', in Mann. DL, Zipes, DP \& Libby, P (eds), Braunwald's Heart Disease: A Textbook of Cardiovascular Medicine, 10th edition, elsevier Saunders, Philadelphia, 2015;179-60.

28. 28. Hagström E, Ahlström T, Arnlöv J, Larsson A, Melhus H, Hellman $\mathrm{P}$, et al. Parathyroid hormone and calcium are independently associated with subclinical vascular disease in a community-based cohort. Atherosclerosis. 2015;238:420-26.

29. 29. Nuzzo V, Tauchmanovà L, Fonderico F, Trotta R, Fittipaldi MR, Fontana D, et al. Increased intima-media thickness of the carotid artery wall, normal blood pressure profile and normal left ventricular mass in subjects with primary hyperparathyroidism. Eur J Endocrinol. 2002;147:453-59.

30. 30. Schillaci G, Pucci G, Pirro M, Monacelli M, Scarponi AM, Manfredelli MR, et al. Large-artery stiffness: a reversible marker of cardiovascular risk in primary hyperparathyroidism. Atherosclerosis. 2011;218:96-101.

31. Kamycheva E, Sundsfjord J, Jorde R. Serum parathyroid hormone levels predict coronary heart disease: the Tromso Study. Eur J Cardiovasc Prev Rehabil. 2004;11:69-74.

32. Hagström E, Michaëlsson K, Melhus H, Hansen T, Ahlström $\mathrm{H}$, Johansson L, et al. Plasma parathyroid hormone is associated with subclinical and clinical atherosclerotic disease in 2 community-based cohorts. Arterioscler Thromb Vasc Biol. 2014;34:1567-73.

33. Perkovic V, Hewitson TD, Kelynack KJ, Martic M, Tait MG, Becker GJ. Parathyroid hormone has a prosclerotic effect on vascular smooth muscle cells. Kidney Blood Press Res. 2003;26:27-33.

34. 11. Amann K, Törnig J, Flechtenmacher C, Nabokov A, Mall G, Ritz E. Blood-pressure-independent wall thickening of intramyocardial arterioles in experimental uraemia: evidence for a permissive action of PTH. Nephrol Dial Transplant. 1995; 10:2043-48.

35. 12. Saleh FN, Schirmer H, Sundsfjord J, Jorde R. Parathyroid hormone and left ventricular hypertrophy. Eur Heart J. 2003;24:2054-60.

36. 13. Bukoski RD, Ishibashi K, Bian K. Vascular actions of the calcium regulating hormones. Semin Nephrol. 1995;15: 536-49.

37. 14. Kosch M, Hausberg M, Vormbrock K, Kisters K, Rahn $\mathrm{KH}$, Barenbrock M. Studies on flow-mediated vasodilation and intima-media thickness of the brachial artery in patients with primary hyperparathyroidism. Am J Hypertens. 2000;13:759-64.

38. Shekarkhar S, Foroughi M, Moatamedi M, Gachkar L. The association of serum parathyroid hormone and severity of coronary artery diseases. Coron Artery Dis. 2014;25: $339-42$.

39. Altay H, Zorlu A, Binici S, Bilgi M, Y1lmaz MB, Çölkesen Y, et al. Relation of serum parathyroid hormone level to severity of heart failure. Am J Cardiol. 2012;109:252-6.

40. Öztürk D, Özcan Çelebi O, Ulusoy FV, Aydodu S. The relationship between serum parathormone levels and the severity of coronary artery disease. Turk Kardiyol Dern Ars. 2012;40:589-94.

41. Reis JP, von Mühlen D, Michos ED, Miller ER, 3rd, Appel LJ, Araneta MR, et al. Serum Vitamin D, parathyroid hormone levels and carotid atherosclerosis. Atherosclerosis. 2009; 207:585-90.

42. 29. Rashid G, Bernheim J, Green J, Benchetrit S. Parathyroid hormone stimulates endothelial expression of atherosclerotic parameters through protein kinase pathways. Am J Physiol Renal Physiol. 2007;292:1215-8.

43. Shekarkhar S, Foroughi M, Moatamedi M, Gachkar L. The association of serum parathyroid hormone and severity of coronary artery diseases. Coron Artery Dis. 2014;25: $339-42$.

44. Deo R, Katz R, Shlipak MG, Sotoodehnia N, Psaty BM, Sarnak MJ, et al. Vitamin D, parathyroid hormone and sudden cardiac death: results from the Cardiovascular Health Study. Hypertension. 2011;58:1021-8. 